\title{
New concepts of cellular fatty acid uptake: role of fatty acid transport proteins and of caveolae
}

\author{
Jürgen Pohl, Axel Ring, Robert Ehehalt, Thomas Herrmann and Wolfgang Stremmel* \\ Departments of Gastroenterology and Internal Medicine, University of Heidelberg, Bergheimer Str. 58, \\ 69115 Heidelberg, Germany
}

\begin{abstract}
Efficient uptake and channelling of long-chain fatty acids (LCFA) are critical cell functions. Evidence is emerging that proteins are important mediators of LCFA-trafficking into cells and various proteins have been suggested to be involved in this process. Amongst these proteins is a family of membrane-associated proteins termed fatty acid transport proteins (FATP). So far six members of this family, designated FATP 1-6, have been characterized. FATP 1, 2 and 6 show a highly-conserved AMP-binding region that participates in the activation of very-long-chain fatty acids (VLCFA) to form their acyl-CoA derivatives. The mechanisms by which FATP mediate LCFA uptake are not well understood, but several studies provide evidence that uptake of LCFA across cellular membranes is closely linked to acyl-CoA synthetase activity. It is proposed that FATP indirectly enhance LCFA uptake by activating VLCFA to their CoA esters, which are required to maintain the typical structure of lipid rafts in cellular membranes. Recent work has shown that the structural integrity of lipid rafts is essential for cellular LCFA uptake. This effect might be exerted by proteins, e.g. caveolin-1 and FAT/CD36, that use lipid rafts as platforms and bind or transport LCFA. The proposed molecular mechanisms await further experimental investigation.
\end{abstract}

Fatty acid transport proteins: Lipid rafts: Caveolae: FAT/CD36: Caveolin-1: Acyl-CoA

\section{Function and transport of long-chain fatty acids}

Long-chain fatty acids (LCFA) are key metabolites associated with energy generation and storage, and are precursors for the biosynthesis of complex membrane lipids. The acyl chain composition of these lipids determines the overall membrane structure and function. LCFA are almost completely absorbed by enterocytes in the jejunum and ileum and enter the lymphatic system and bloodstream as chylomicrons. Capillary-bound lipoprotein lipase, produced by liver, heart, adipose and other tissues, catalyses the release of LCFA from lipoproteins, of which the vast majority is immediately bound to albumin. LCFA, as LCFA-albumin, are transported in the bloodstream and taken up by the various tissues of the body. It was initially believed that LCFA enter cells merely by diffusion through the phospholipid bilayer. However, there is now sufficient evidence to suggest that, in addition to this diffusion component, physiological uptake of LCFA across cell membranes is a saturable and inhibitable process, indicative of protein-mediated transport.

\section{The fatty acid transport protein family}

Indeed, an expression-cloning approach identified an adipocyte protein (termed fatty acid transport protein; FATP) that, when expressed in mammalian cells, facilitates the uptake of LCFA (Schaffer \& Lodish, 1994). This protein is expressed in cells with high-level LCFA uptake for metabolism and storage. FATP has a distinct membrane topology and shows a single transmembrane domain and multiple membrane-associated domains peripherally associated with the inner leaflet of the plasma membrane (Lewis et al. 2001; Fig. 1). Meanwhile, a family of FATP with six members (FATP1-6) has been identified (Hirsch et al. 1998; Gimeno et al. 2003), and the FATP originally described was renamed FATP1. The FATP family shows highly-conserved AMP-binding regions, representing the catalytic region of the protein. Indeed, acyl-CoA synthetase activity with a particular preference for very-longchain fatty acids (VLCFA) was demonstrated for FATP1, 2, and 4 (Berger et al. 1998; Coe et al. 1999; Steinberg et al. 1999a,b; Herrmann et al. 2001). Interestingly, in 


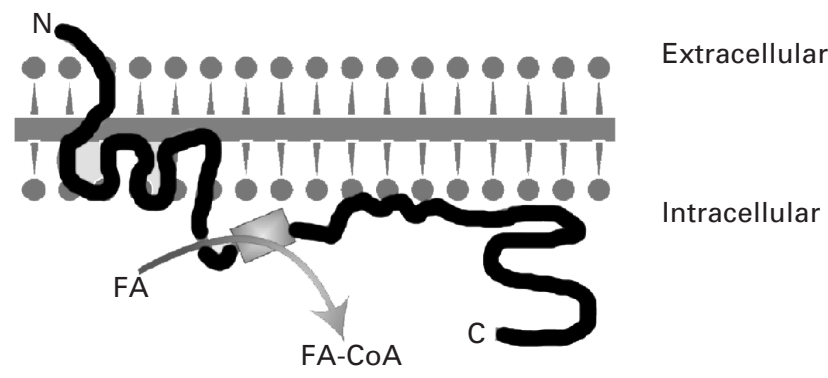

Fig. 1. Schematic representation of the membrane topology of fatty acid (FA) transport protein.

mammalian organisms, FATP isoforms have a distinct tissue-specific distribution (Frohnert \& Bernlohr, 2000). While FATP1 is found in adipose tissue and in the heart, it is absent in the liver. In contrast, FATP2 and FATP5 are almost exclusively found in the liver and FATP4 is the only FATP expressed in the intestine (for review, see Stahl et al. 2001). FATP family members are not cytosolic proteins but rather integral membrane proteins whose subcellular localization is highly variable. FATP1 has been shown to be located on the plasma membrane and in small vesicles distributed throughout the cytoplasm (Schaffer \& Lodish, 1994), and insulin stimulation results in the translocation of FATP1 from an intracellular perinuclear compartment to the plasma membrane (Stahl et al. 2002). The findings of other studies have suggested that FATP4 resides in endomembranes in the vicinity of the plasma membrane (Stahl et al. 1999; Herrmann et al. 2003).

The mechanisms by which FATP mediate LCFA uptake are not well understood. Specific binding sites for LCFA within the FATP structure have not yet been identified and FATP do not show any obvious similarities to other transporter families. However, there is evidence that uptake of LCFA across cellular membranes might be linked to FATP acyl-CoA synthetase activity (Coe et al. 1999). To explain both transport and very-long-chain acyl-CoA synthetase activity for FATP, it is proposed that FATP indirectly facilitate cellular LCFA uptake by activation of VLCFA that are incorporated into specialized microdomains termed lipid rafts.

\section{Involvement of lipid rafts and caveolae in long-chain fatty acids uptake}

VLCFA are thought to be essential components of lipid rafts, which represent small platforms $(30-50 \mathrm{~nm}$ diameter) of proteins and lipids within cellular membranes and play an important role in membrane compartmentation, membrane sorting and trafficking. Caveolae represent specialized microdomains formed through coalescence of raft domains and recruitment of caveolin-1, their major structural protein (Fig. 2). The raft membrane is composed of sphingolipids and cholesterol in the exoplasmic leaflet, linked to glycerophospholipids and cholesterol in the cytoplasmic leaflet of the lipid bilayer (Simons \& Ehehalt, 2002). It is not clear how the inner leaflet is coupled to the outer leaflet, but one possibility is that VLCFA substituents of sphingolipids extend from the outer to the inner leaflet where they interdigitate with the saturated side chains of

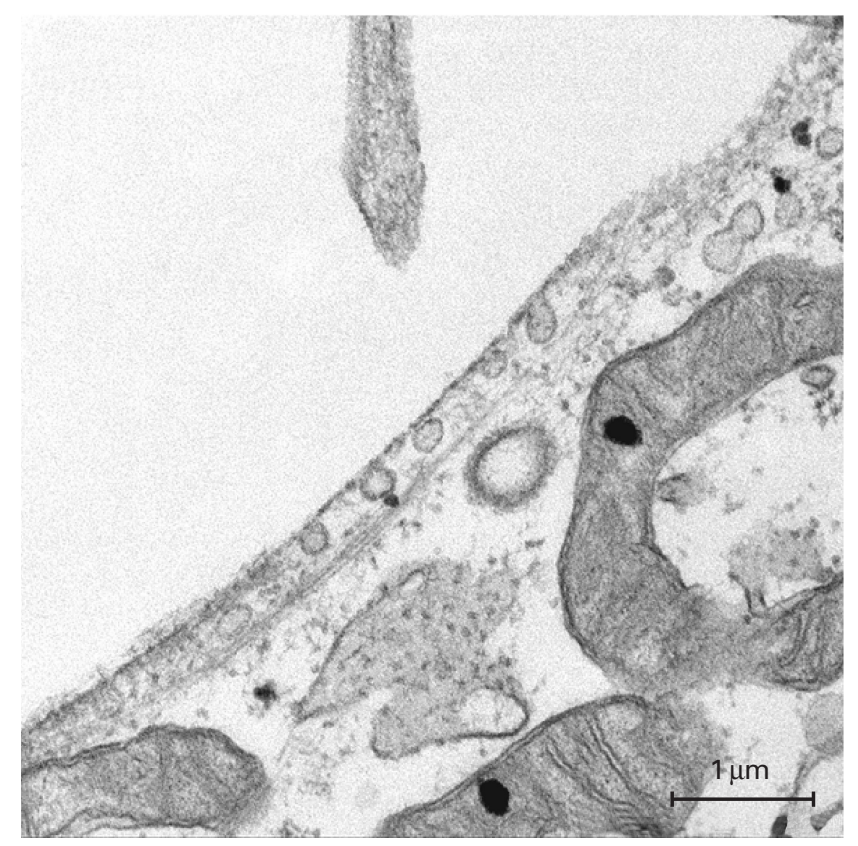

Fig. 2. Surface segment of 3T3-L1 adipocytes analysed by electron microscopy. The plasma membrane shows typical flaskshaped invaginations termed caveolae.

glycerophospholipids and establish a close association (Rietveld \& Simons, 1998). Thus, by activating the conversion of VLCFA to their acyl-CoA derivatives FATP 1, 2 and 4 might play an important role in the sphingolipiddependent membrane bilayer interdigitation process within raft microdomains.

The findings that a major protein associated with fatty acid uptake, FAT/CD36 (Abumrad et al. 1993), is preferentially located in the caveolae at the plasma membrane level (Kolleck et al. 2002), and that caveolin-1 binding with fatty acids is saturable (Trigatti et al. 1999) stimulated interest in the possibility that caveolae might have a function in lipid transport. Indeed, in previous studies (Pohl et al. 2002; Ring et al. 2002) it was shown that caveolae are associated with the overall process of cellular uptake of LCFA. When caveolin-1-green fluorescence protein was expressed in HepG2 cells and exposed to the fluorescent LCFA derivative 12-(N-methyl)-N-[(7-nitrobenz-2-oxa-1, 3-diazol-4-yl)amino] stearate, there was marked co-localization of 12-(N-methyl)-N-[(7-nitrobenz-2-oxa-1,3-diazol4-yl)amino] stearate with green fluorescence protein. Dissociation of rafts by cholesterol depletion (Pohl et al. 2002) or transfection of the dominant-negative caveolin mutant $\mathrm{Cav}^{\mathrm{DGV}}$ (J Pohl, A Ring, R Ehehalt, H SchulzeBergkamen, A Schad, P Verkade and W Stremmel, unpublished results) markedly reduced internalization of LCFA.

These studies might explain recent findings with caveolin-1 knock-out mice. Although these mice lack caveolae in all non-muscle tissues, at first they appeared to be quite healthy (Razani et al. 2001). However, as the mice were studied further it became apparent that transport of glycosylphosphatidylinositol-anchored proteins to the cell surface was inefficient, consistent with a role for caveolin in the transport of these lipid raft-associated proteins to the plasma membrane (Sotgia et al. 2002). The most important 


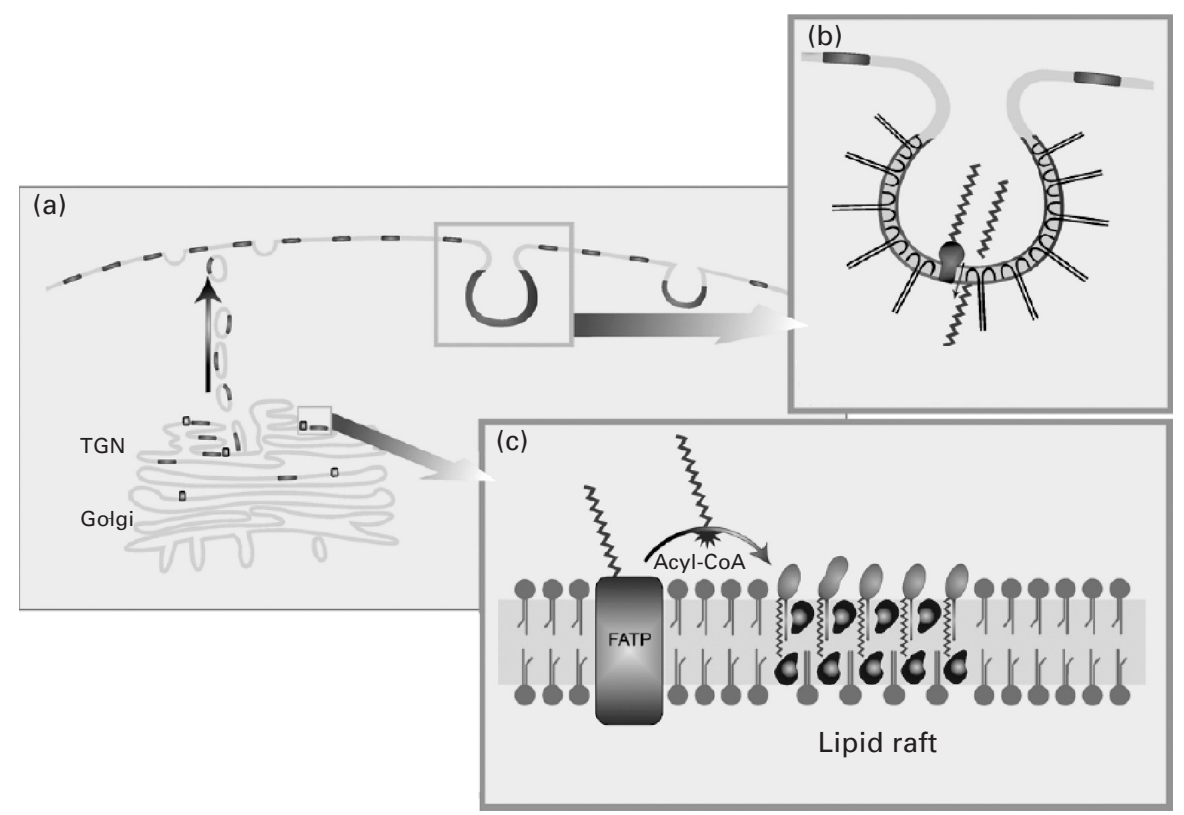

Fig. 3. Proposed model for cellular fatty acid uptake. Fatty acid transport protein (FATP, $\square$ ), stabilization of lipid rafts $(-)$ by acylated very-long-chain fatty acids (VLCFA) and fatty acid translocase (FAT)/CD36 incorporation into lipid rafts could cooperate to facilitate efficient long-chain fatty acid (LCFA) uptake. Intracellular VLCFA are esterified to their acyl-CoA and thereafter bound to sphingolipids in the outer leaflet of the biomembrane of lipid rafts. This process might indirectly facilitate 'on-demand' recruitment of FAT/CD36 from an intracellular compartment to lipid rafts located on the plasma membrane and result in augmented uptake of LCFA. TGN, trans-Golgi network; ( $)$ ), caveolins; (๕)), fat; (mm), fatty acid; (१), phospholipid; (C), cholesterol; (1), sphingolipid.

phenotype of the knock-out mice was a resistance to dietinduced obesity (Razani et al. 2002). Although insulin, glucose and cholesterol levels were normal, caveolin-1 null mice showed a dramatic increase in serum triacylglycerols and NEFA levels, especially in the postprandial state (Razani et al. 2002).

Taken together, these findings strengthen the idea that, in certain cell types, caveolae might have a primary role in regulating the lipid balance by stimulating LCFA uptake. This effect might be exerted by 'on demand' recruitment of LCFA transport proteins to lipid rafts, either from other membrane domains or from intracellular compartments.

\section{A concept for long-chain fatty acid uptake}

It is hypothesized that the increase in cellular fatty acid uptake observed in cells overexpressing FATP represents an indirect event involving stimulation of lipid raft constitution and stabilization (Fig. 3). Thus, VLCFA taken up into cells via the known membrane transporters (fatty acid translocase/CD36, plasma membrane fatty acid-binding protein) might be activated by FATP to form very-longchain acyl-CoA and could be incorporated into the sphingolipids in lipid rafts. These microdomains are transported and integrated into the plasma membrane where they serve as platforms for proteins involved in the binding and uptake of LCFA, e.g. fatty acid translocase/CD36 and caveolin-1.

At the plasma membrane level, uptake of LCFA might include the following subsequent steps: (a) dissociation of fatty acids bound to albumin in the vicinity of the plasma membrane through interaction with membrane-associated fatty acid-binding proteins (e.g. fatty acid translocase/ CD36); (b) translocation via a membrane fatty acid carrier or protonization of LCFA followed by flip-flop movement across the membrane bilayer according to the concentration gradient; (c) binding to caveolin-1 at the inner leaflet of the lipid bilayer. Here, caveolin-1 might accumulate LCFA at the inner leaflet and present LCFA to cytoplasmic fatty acid-binding proteins for further shuttling of LCFA to different organelles. Alternatively, caveolin-1 may itself serve as a carrier for intracellular transport.

Although this model is in accordance with our current understanding of LCFA uptake into cells, it is purely hypothetical and much work remains to be done to determine the molecular mechanism of FATP functions in LCFA uptake.

\section{Acknowledgements}

Supported by grant STR 216/11-1 from the Deutsche Forschungsgemeinschaft and generous gifts from the Dietmar Hopp Foundation.

\section{References}

Abumrad NA, El-Maghrabi MR, Amri EZ, Lopez E \& Grimaldi PA (1993) Cloning of rat adipocyte membrane-protein implicated in binding or transport of long chain fatty acids 
that is induced during preadipocyte differentiation: homology with human CD 36. Journal of Biological Chemistry 268, 17665-17668.

Berger J, Truppe C, Neumann H \& Forss-Petter S (1998) A novel relative of the very-long-chain acyl-CoA synthetase and fatty acid transporter protein genes with a distinct expression pattern. Biochemical and Biophysical Research Communications 247, 255-260.

Coe NR, Smith AJ, Frohnert BI, Watkins PA \& Bernlohr DA (1999) The fatty acid transport protein (FATP1) is a very long chain acyl-CoA synthetase. Journal of Biological Chemistry 274, 36300-36304.

Frohnert BI \& Bernlohr DA (2000) Regulation of fatty acid transporters in mammalian cells. Progress in Lipid Research 39, 83-107.

Gimeno RE, Ortegon AM, Patel S, Punreddy S, Ge P, Sun Y, Lodish HF \& Stahl A (2003) Characterization of a heartspecific fatty acid transport protein. Journal of Biological Chemistry 278, 16039-16044.

Herrmann T, Buchkremer F, Gosch I, Hall AM, Bernlohr DA \& Stremmel W (2001) Mouse fatty acid transport protein 4 (FATP4): Characterization of the gene and functional assessment as a very long chain acyl-CoA synthetase. Gene 270, $31-40$.

Herrmann T, van der Hoeven F, Gröne HJ, Stewart AF, Langbein L, Kaiser I, Liebisch G, Gosch I, Buchkremer F, Drobnik W, Schmitz G \& Stremmel W (2003) Mice with targeted disruption of the fatty acid transport protein 4 (Fatp 4, Slc27a4) gene show features of lethal restrictive dermopathy. Journal of Cell Biology 161, 1105-1115.

Hirsch D, Stahl A \& Lodish HF (1998) A family of fatty acid transporters conserved from mycobacterium to man. Proceedings of the National Academy of Sciences USA 95, 8625-8629.

Kolleck I, Guthmann F, Ladhoff AM, Tandon NN, Schlame M \& Rüstow B (2002) Cellular cholesterol stimulates uptake of palmitate by redistribution of fatty acid translocase in type II pneumocytes. Biochemistry 41, 6369-6375.

Lewis SE, Listenberger LL, Ory DS \& Schaffer JE (2001) Membrane topology of the murine fatty acid transport protein 1. Journal of Biological Chemistry 276, 37042-37050.

Pohl J, Ring A \& Stremmel W (2002) Uptake of long-chain fatty acids in HepG2 cells involves caveolae: analysis of a novel pathway. Journal of Lipid Research 43, 1390-1399.

Razani B, Engelman JA, Wang XB, Schubert W, Zhang XL, Marks CB et al. (2001) Caveolin-1 null mice are viable but show evidence of hyperproliferative and vascular abnormalities. Journal of Biological Chemistry 276, 38121-38138.
Razani B, Combs TP, Wang XB, Frank PG, Park DS, Russell RG, Li M, Tang B, Jelicks LA, Scherer PE \& Lisanti MP (2002) Caveolin-1 deficient mice are lean, resistant to dietinduced obesity, and show hypertriglyceridemia with adipocyte abnormalities. Journal of Biological Chemistry 277, 8635-8647.

Rietveld A \& Simons K (1998) The differential miscibility of lipids as the basis for the formation of functional membrane rafts. Biochimica et Biophysica Acta 1376, 467-479.

Ring A, Pohl J, Völkl A \& Stremmel W (2002) Evidence for vesicles that mediate long-chain fatty acid uptake by human microvascular endothelial cells. Journal of Lipid Research 43, 20095-20104.

Schaffer JE \& Lodish HF (1994) Expression cloning and characterization of a novel adipocyte long chain fatty acid transport protein. Cell 79, 427-436.

Simons K \& Ehehalt R (2002) Cholesterol, lipid rafts, and disease. Journal of Clinical Investigation 110, 597-603.

Sotgia F, Razani B, Bonuccelli G, Schubert W, Battista M, Lee H, Capozza F, Schubert AL, Minetti C, Buckley JT \& Lisanti MP (2002) Intracellular retention of glycosylphosphatidyl inositol-linked proteins in caveolin-deficient cells. Molecular and Cellular Biology 22, 3905-3926.

Stahl A, Evans JG, Pattel S, Hirsch D \& Lodish HF (2002) Insulin causes fatty acid transport protein translocation and enhanced fatty acid uptake in adipocytes. Developmental Cell 2, 477-488.

Stahl A, Gimeno RE, Tartaglia LA \& Lodish HF (2001) Fatty acid transport proteins: a current view of a growing family. Trends in Endocrinology and Metabolism 12, 266-273.

Stahl A, Hirsch DJ, Gimeno RE, Punreddy S, Ge P, Watson N, Patel S, Kotler M, Raimondi A, Tartaglia LA \& Lodish HF (1999) Identification of the major intestinal fatty acid transport protein. Molecular Cell 3, 299-308.

Steinberg SJ, Wang SJ, Kim DG, Mihalik SJ \& Watkins PA (1999a) Human very-long-chain acyl-CoA synthetase. Cloning, topography, and relevance to branched-chain fatty acid metabolism. Biochemical and Biophysical Research Communications 257, 615-621.

Steinberg SJ, Wang SJ, McGuinness MC \& Watkins PA (1999b) Human liver-specific very-long-chain acyl-coenzyme A synthetase: cDNA cloning and characterization of a second enzymatically active protein. Molecular Genetics and Metabolism 68, 32-42.

Trigatti BL, Anderson RG \& Gerber GE (1999) Identification of caveolin-1 as a fatty acid binding protein. Biochemical and Biophysical Research Communications 255, 34-39. 